\title{
Cu Doped TiO2 Thin Films Fabricated by Simple SPD Technique
}

\author{
G. Jeevarani Thangama, J. Jebaraj Devadasana*, P. Shanthini Gracea \\ P.G. Department of Physics, Pope's College, Sawyerpuram-628253, Tamilnadu, India.
}

\begin{abstract}
Cu}$-doped TiO2 thin films were synthesised by spray pyrolysis method onto glass substrates at a substrate temperature of about $3500 \mathrm{c}$ and annealed at $5000 \mathrm{c}$ for 1 hour. The annealed films were characterised by $x$-ray diffraction, scanning electron microscope and also subjected to photoluminescence studies in order to characterize the structural property, surface morphology and to determine trapping of charge carriers in semiconductors. The diffraction peaks from XRD were index to anatase phase for both undoped and doped TiO2 thin films. The results showed that at doping the intensity of (101) plane decreased which may be due to mobility of titanium and oxygen atoms which leds to reduction in the nucleation of crystallisation phase of anatase TiO2. The SEM structure revealed a uniform and evenly distributed grains across the substrate surface. Photoluminescence studies revealed a low PL intensity for doped films and is attributed to the reduction of oxygen vacancies.
\end{abstract}

Keywords: TiO2, XRD, SEM, EDAX, PL

\section{Introduction}

Thin film research has been widely expanded due to the increasing demands for microelectronics and microstructural components in different branches of science and technology. Transparent and conductive oxides are extensively used for variety of applications. Among many oxides, Titanium dioxide has unique characteristics and $\mathrm{TiO} 2$ thin films are regarded as promising functional materials for the potential applications such as gas sensors, microelectronics, photocatalytic, photovoltaic devices and ultraviolet blockers (1-3). Titanium dioxide ( $\mathrm{TiO} 2)$ possess a number of attractive properties such as its wide bandgap (3.2eV) for anatase, non toxicity, transparency in the visible light, high refractivity and dielectric constant. It is an n-type semiconductor material with high chemical stability and good performance in its response rate. Titanium dioxide is considered to be one of the most important photo catalytic material and currently there exist a better understanding and improvement of catalytic reactions which is a main driving force for surface investigations of $\mathrm{TiO} 2$. From various metals employed for improving the properties of $\mathrm{TiO} 2$, the doping of $\mathrm{TiO} 2$ with definite metal ions is found to be simple, inexpensive and effective way of preparing $\mathrm{TiO} 2$ with consistent properties respectively. Among the elements doped so far $\mathrm{Cu}$ has been considered to be important owing to narrow band gap energies of its oxides (5). The $\mathrm{Cu}$ doping has been found to replace certain Ti4+ ions in substitutional sites of $\mathrm{TiO} 2$ and to cause the incorporation of $\mathrm{Cu}$ on the surface and into the surface and into the interstitial sites of $\mathrm{TiO} 2$. There are several $\mathrm{TiO} 2$ thin film deposition techniques including metal organic chemical vapour deposition(6), sol-gel(7),pulsed laser deposition (8),ion beam enhanced deposition(9) and electrophoreic deposition (10).In our report $\mathrm{Cu}$ doped $\mathrm{TiO} 2$ thin films were prepared by a homogenous method of spray pyrolysis technique and calcined at different temperatures. The films were then characterized by various techniques and the results of X-ray diffraction,SEM analysis and photoluminescence has been discussed. The simple spray pyrolysis technique chosen here has its own inherent advantages overall conventional processes. By this method we can produce particles of various morphologies. Depending on application proper selection of precursors, additives and heat sources is needed to prepare thin films.

\subsection{Material Synthesis}

\section{Materials and Methods}

The reference $\mathrm{TiO} 2$ and $\mathrm{Cu}$ doped $\mathrm{TiO} 2$ thin films were prepared by spray pyrolysis deposition method on glass substrates. The precursor solution consist of titanium (iv) butaoxide, cupric chloride (only in cu doped $\mathrm{TiO} 2$ ), hydrochloric acid and deionized water. In order to achieve a good adhesion of the coating material onto the substrate special attention was paid to the preparation of substrates. A standard procedure was adopted for the cleaning of the substrate prior to the spray process. The directly derived films were amorphous, thus they were annealed at $5000 \mathrm{C}$ for 1 hour to remove the organic solvents and to achieve crystallization.

\subsection{Material Characterization:}

The crystal structure was analysed by XRD and the XRD patterns were collected in $2 \theta$ range from 200 to 800 .using $\mathrm{Cu} \mathrm{K \alpha}$ radition performed by X-ray diffractometer. The surface morphology study was performed National Conference on Current Advancements in Physics $3^{\text {rd }} \& 4^{\text {th }}$ February $2017 \quad 57 \mid$ Page Department of Physics, St. John's College, Palayamkottai-627 002, Tamilnadu, India. DOI 10.9790/4861-17002035760 
using scanning electron microscope. The composition of the films were determined by energy dispersive X-ray spectroscopy.Photoluminescence studies were also carried out.

\subsection{Structure and Analysis}

\section{Results and Discussion}

$\mathrm{X}$-ray diffraction pattern of undoped and $\mathrm{Cu}$ doped titanium dioxide thin films are shown in fig 3.1.The films are polycrystalline and fit well with tetragonal crystal structure. The diffraction peaks from the XRD pattern is in agreement with the JCPDS card no 21-1272. Fig 3.2 represents the copper doped titanium dioxide thin films.The diffraction peaks were indexed to anatase planes and $\mathrm{Cu}$ relative peaks were not observed implying that $\mathrm{Cu}$ was doped into $\mathrm{TiO} 2$ lattice. At doping of $\mathrm{Cu}$ the intensity of (101) plane decreases which may be due to decrease in the mobility of titanium and oxygen atoms which led to the reduction in the nucleation of crystallization phase of anatase $\mathrm{TiO} 2$. The crystallite size (d) of the sample was calculated from full width at half maximum (FWHM) of the (101) peak of anatase TiO2 by Debye Scherrer equation.

$$
\mathrm{d}=\mathrm{k} \lambda / \beta \cos \theta
$$

where $\mathrm{d}$ represents the crystallite size, $\lambda$ represents the wavelength of incident $\mathrm{x}$-ray, $\beta$ represents the FWHM of the diffraction peak,$\theta$ represents the scaterring angle. The crystallite size decrease with increase in $\mathrm{Cu}$ content which is attributed to the stress produced by the ionic difference of $\mathrm{Cu} 2+$ and $\mathrm{Ti} 4+$ The lattice strain is calculated using the relation

$$
€=\beta \cos \theta / 4
$$

The dislocation density is defined as the length of dislocation lines per unit volume of the crystal and was estimated from the following relation using the simple approach of Williamson and smallmen. The value of dislocation density $(\delta)$ is calculated using the relation.

$$
\delta=1 / \mathrm{D} 2
$$

It is observed that as the doping concentration increases the dislocation density is increased because of the difference in ionic radii of $\mathrm{Ti} 4+$ and $\mathrm{Cu} 2+$. The lattice constants were calculated for $\mathrm{TiO} 2$ thin films and it is found that the ' $a$ ' and 'c' values are in concordance with the standard values $(a=3.785 \mathrm{~nm}$ and $c=9.513 \mathrm{~nm})$ these indicate that the quality of $\mathrm{TiO} 2$ films is good and crystalline in nature. The lattice parameter of the doped films ' $a$ ' and ' $c$ ' is less then undoped which is a strong indication of stress in the films.

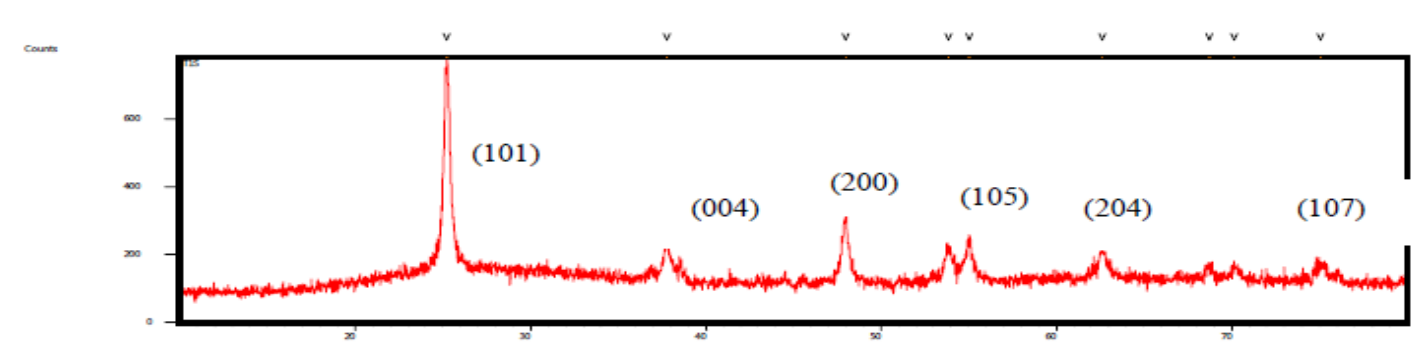

Fig 3.1: Undoped $\mathrm{TiO}_{2}$ Thin film
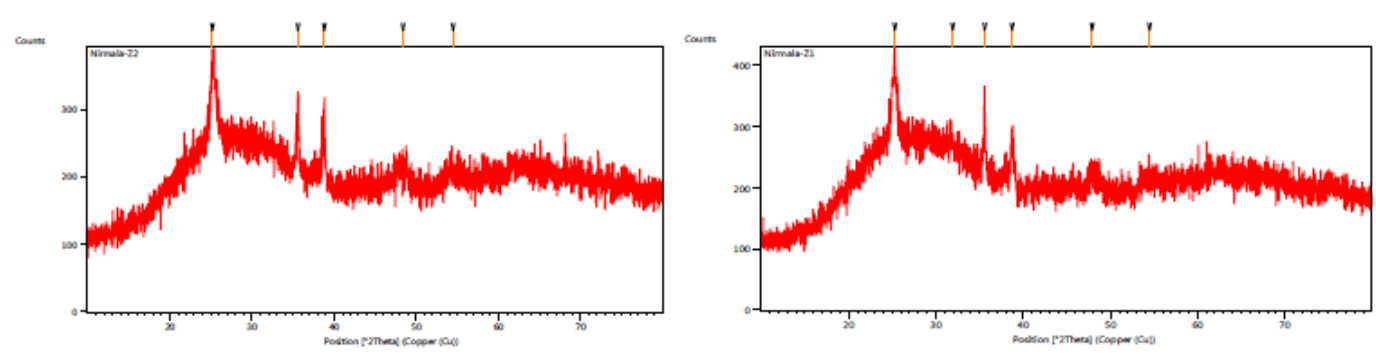

Fig 3.2: Doped $\mathrm{TiO}_{2}$ Thin film 
Cu Doped TiO2 Thin Films Fabricated by Simple SPD Technique.

\begin{tabular}{|l|l|l|l|l|l|}
\hline \multicolumn{1}{|c|}{ Films } & \multicolumn{1}{|c|}{$\begin{array}{c}\text { Substrate } \\
\text { temperature }(C)\end{array}$} & $\begin{array}{c}\text { Crystallite } \\
\text { Size }(\mathrm{nm})\end{array}$ & \multicolumn{1}{|c|}{ Strain $(€)$} & $\begin{array}{c}\text { Dislocation } \\
\text { density }(\delta) \text { Lines } / \mathrm{m}^{2}\end{array}$ & \multicolumn{1}{|c|}{ Structure } \\
\hline Undoped & $350^{\circ} \mathrm{c}$ & $47 \mathrm{~nm}$ & 7.1 & .0064 & Tetragonal \\
\hline Cu-doped & $350^{0} \mathrm{c}$ & $\mathbf{3 1 . 2 \mathrm { nm }}$ & $\mathbf{3 . 2}$ & .0237 & Tetragonal \\
\hline
\end{tabular}
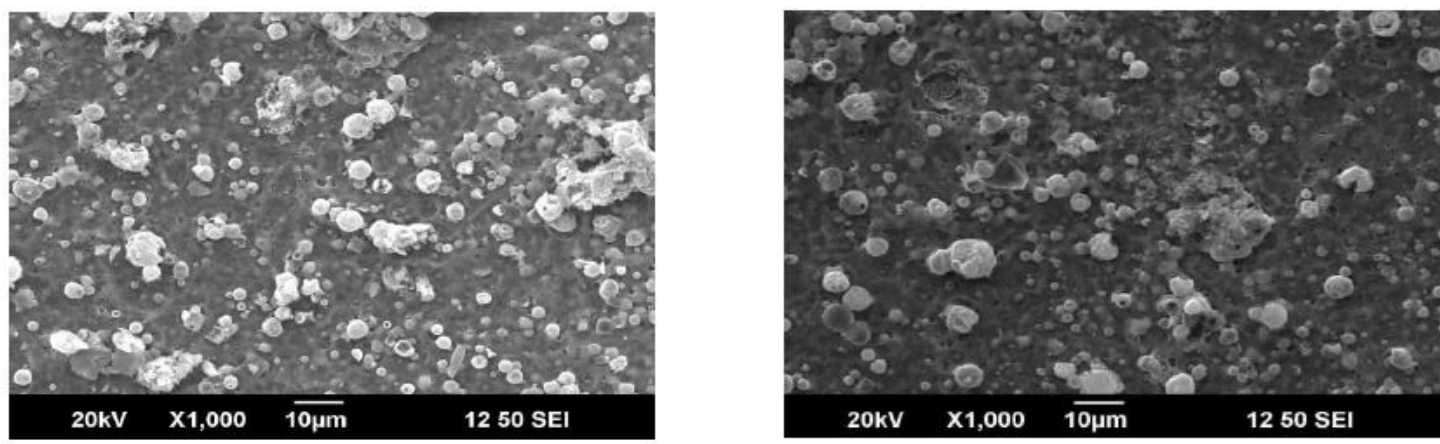

Fig 3.3: Undoped $\mathrm{TiO}_{2}$ Thin film
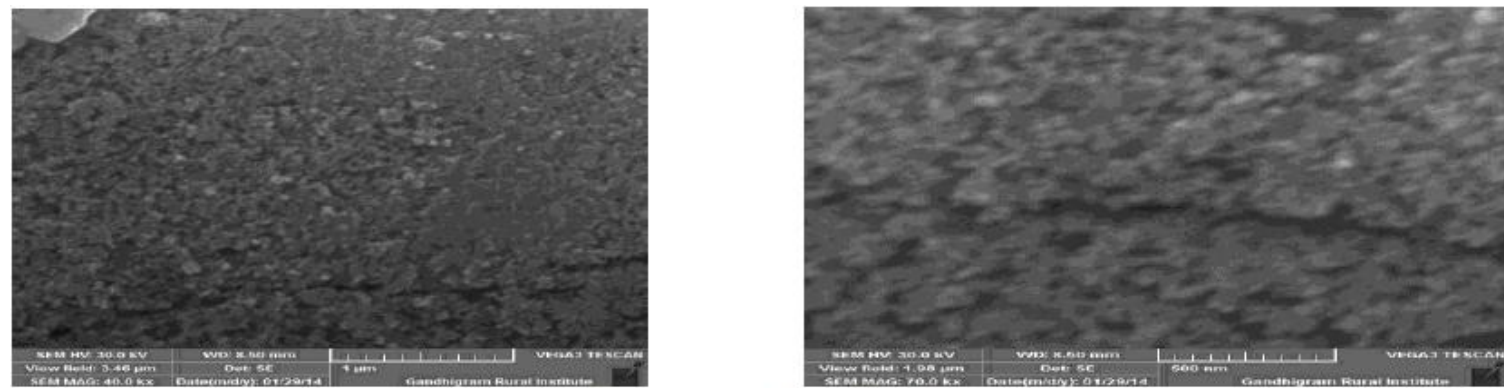

Fig 3.4: Doped $\mathrm{TiO}_{2}$ Thin film

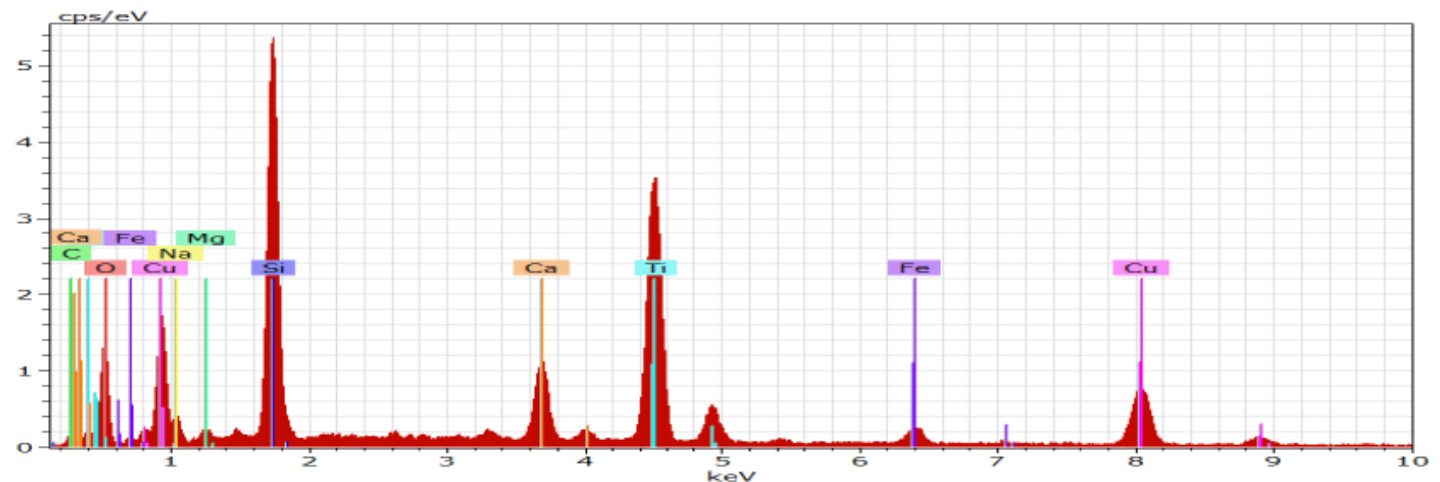

Fig 3.5: EDAX of $\mathrm{Cu}$ doped $\mathrm{TiO}_{2}$ Thin film

The morphology of the thin films is analysed by scanning electron microscope. Fig $3.3 \& 3.4$ shows the SEM micrographs of pristine $\mathrm{TiO} 2$ and $\mathrm{Cu}$ doped $\mathrm{TiO} 2$ on glass substrates deposited at an optimized temperature of 3500C. The SEM micrographs indicated that the film is well and evenly distributed across the substrate surface as clearly shown in figure. The grains have regular structure with layers and the layers are described as overlapping flakes. Fig 3.5 shows the EDAX spectrum of copper doped TiO2 thin films. The EDAX spectrum also confirmed the presence of copper, titanium and oxygen as the elements present in the film.

\subsection{Photoluminescence (PL):}

Photoluminescence emission spectra can be used to investigate the efficiency of charge trapping, immigration transfer and to understand the fate of electron hole pairs in semiconductor particles since PL emission results from the recombination of charge carriers. Fig $3.6 \& 3.7$ shows the PL emission spectra of both pure and copper doped $\mathrm{TiO} 2$ thin film measured from 200 to $900 \mathrm{~nm}$ 


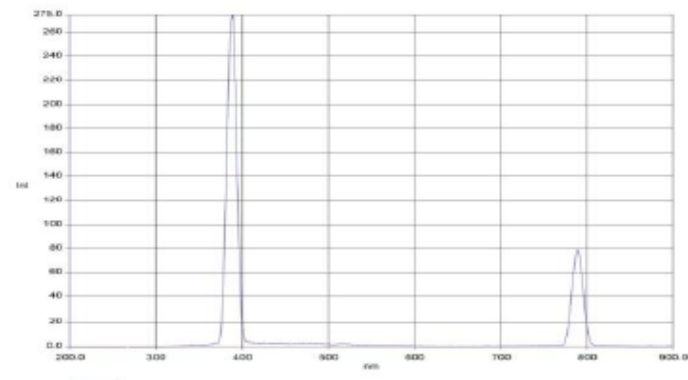

Fig 3.6: Undoped $\mathrm{TiO}_{2}$

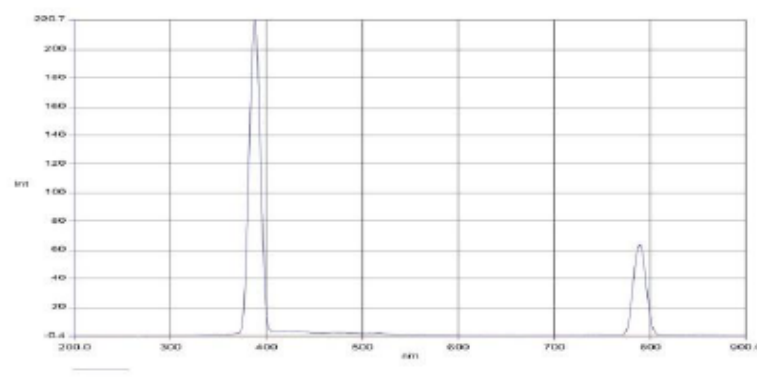

Fig 3.7: Doped $\mathrm{TiO}_{2}$

PL intensities were quite sensitive to metal doping and the peak position of pure $\mathrm{TiO} 2$ were consistent with copper doped TiO2.Fig 3.6 \& Fig 3.7 shows the intensity of photoluminescence spectrum of the pristine $\mathrm{TiO} 2$ film is much higher than the doped $\mathrm{TiO} 2$ film. The reduction of photoluminescence intensity with the increase in dopant concentration indicates the retardation of recombination process which is probably due to the efficient transfer of charge into highly disperse $\mathrm{Cu}$. A decrease in photo luminescence intensity indicates a lower recombination rate of electron-hole pairs and hence higher separation efficiency.

\section{Conclusion}

The study of undoped and copper doped $\mathrm{TiO} 2$ thin films were prepared by spray pyrolysis technique using precursor solution of titanium (iv) butaoxide. The XRD pattern of deposited films (TiO2 and $\mathrm{Cu}$ doped TiO2) revealed the tetragonal structure with preferential orientation along (101) plane. The SEM study evidenced grains with uniform nature. It also reveals the reduction in size of the particles by addition of the dopant. The PL intensity of the $\mathrm{Cu}$ doped $\mathrm{TiO} 2$ film decreased and it was attributed to the retardation of recombination process.

\section{References}

[1]. N.H.Sun, X.C.Bai, M.W.Li,B.L.Yao Adv.Mater.Res 210, 2012, 482-484.

[2]. Tae Young Lee,P.S.Alegaonkar,Ji-Beom Yoo "Fabrication of dye syntesised solar cell using TiO2 coated carbon nanotubes ". Thin solid films 515,2007,5131-5135.

[3]. Y.Y.Kee,S.S.Tan,T.K.Yong,C.H.Nee,S.S.Yap,T.Y.Tou,G.Saffran,Z.E.horvath,J.P.MoscatelloY.K.Yap Nanotechnology 23,2012.

[4]. N.R.Mathews,M.A.C.Jacome, E.R.Morales,J.A.T.Antoniom "Structural and Spectroscopic study of Fe doped TiO2 thin films for applications in photocatalysis. "Physics status Solids Vol6,April 2009, 219-223.

[5]. H.A.Foster,D.W.sheeel,P.Sheel,P.Evans,S.Vargnese,N.Rutschke,H.M.Yares,J.Photochem,PhotobiolA:Chem $216,2010$.

[6]. N.Rausch,E.P.Burte,Jurnal of Electrochemical society 140,1993,145-149.

[7]. N.Ozer,H.Demiryont,J.H.Simmons, Applied optics 30,1991,3661-3666.

[8]. Lei Zhao, Qing Jiang,Jianshe Lian Applied surface science 254,2008,4620-4625.

[9]. JianZhong Zhu, Congxin Ren, Guoliang Chen,Chuying Yu,Jial Wu,Haicuan Mu sensors and Actuators B (32), 1996,209-213.

[10]. J.A.Byrne,B.R.Eggins,S.Linquette Mailley P.S.M.Dunlop,Analyst 123,1998,2007-2012. 